\title{
Interactive Content Using Newtro With A Focus On Hand Interface
}

\author{
Haram Choi and Sanghun Nam* \\ M.A Student, Department of Culture Technology, Changwon National University, \\ 20 Changwondaehak-ro, Uichang-gu, Changwon-si, Gyeongsangnam-do, 51140, KOREA \\ *Corresponding author. Tel.: +82-010-3785-5990; \\ Email address: sanghunnam@ changwon.ac.kr
}

Article History: Received:11 November 2020; Accepted: 27 December 2020; Published online: 05 April 2021

\begin{abstract}
With recent advancements in technology, there has been a tendency to increase the amount of interactive content, e.g., technology combined with cultural content. Based on this, interactive content has been created based on 'Newtro.' In view of the physical interactions between users and content, interactive content with an interface for connecting users and content was set as the object of this study. To apply the physical interaction (considered the most important aspect of the content), 'a button interface' requiring a relatively greater force was used. When the user pressed the button, the video was played on a projector. The physical interactions between users and content were discussed, and an interface for connecting users and contents to thereby apply physical interactions to content was studied. A questionnaire survey was conducted, targeting users who experienced the content, and research results were derived. Therefore, this study is meaningful in that it addresses the physical interactions between users and content and examines the correlation between the physical interface and interaction. Although using a button interface was helpful for the physical interaction, a relatively simple technique was used; this could reduce user interest in the content. Therefore, research on interactivity and interfaces that can promote more active communication between content and users is required.
\end{abstract}

Keywords: Newtro, Interactive contents, Hand interface, Physical interaction, Touch interface.

\section{Introduction}

Considering the recent developments in technology, much of today's content is presented in the form of interactions. Interactivity includes physical and psychological interactions between humans and media, and among humans themselves. Today's content emphasizes the convergence of different fields and is mostly characterized by interactivity. In particular, there is a tendency to increase the interactivity of smart and digital content. In addition to these changes, the amount of interactive content combining digital technology with cultural content is increasing. Interaction has emerged as a major feature of interactive content[1]. In addition, for interactions between the content and a user, an interface (a contact point where the interaction occurs) is necessary. In summary, an interaction is a concept that focuses on an action or activity, and the interface is the point of contact between both systems[2]. 'Newtro' means not only to recreate the past, but also to reinterpret it modernly. Newtro is a concept derived from 'retro' and it is a phenomenon in which generations who have not experienced the past search for contents of the past[3]. In this study, the sociocultural phenomena related to Newtro were analyzed through a literature review. Based on this analysis, research was conducted on interactive content that converged art and technology. Using this interactive content, the psychological stability felt from the use of Newtro was intended to relieve the digital fatigue of the young generation currently living in modern society. Newtro was used as the main visual concept for the content, and the content was reinterpreted by adding a new media interaction element. The interaction between users and contents was the most important consideration for producing interactive content. In particular, most approaches have focused on physical interaction, and to maximize such physical interaction, they employ a button interface by which user can apply physical force and feel with a sense. In the case of an interface where a user's physical force is applied relatively slightly, such as with a touch sensor, the Newtro characteristics cannot be revealed. In the past, most interfaces could be directly touched, and required greater physical force. For example, a 2G phone with a built-in dial requires greater physical force than a smartphone or touch phone and listening to music on an LP that can be touched by a hand requires greater force than playing a digital record. The research question that this study focuses on is: 'Did interactive contents contribute to users' immersion in the interaction between users and content?' Another study, aimed to determine whether an interface requiring relatively greater physical force allowed the user to feel more presence regarding the content, was also conducted. The remainder of this paper is structured as follows. Chapter 2 discusses the definition of Newtro and its sociocultural background. It also addresses the theoretical background for the physical interactions and user interfaces that will be focused on in this study. Chapter 3 describes the interactive content produced based on Chapter 2 and presents an analysis of the technical elements. Finally, in Chapter 4, the results of research on the physical interactivity and interface for interactive content are analyzed based on a survey of users who experienced the content.

*Corresponding author: Sanghun Nam

M.A Student, Department of Culture Technology, Changwon National University,

20 Changwondaehak-ro, Uichang-gu, Changwon-si, Gyeongsangnam-do, 51140, KOREA

Email address: sanghunnam@ changwon.ac.kr 


\section{Materials and methods}

Retro is an abbreviation of 'retrospect,' meaning reminiscence or memory, and has generally denoted reusing special modes, motifs, and techniques from the past, e.g., since the 1960s. French journalists began to use the word 'retro' in the mid-1970s[4, 5]. Recently, it has become a sociocultural phenomenon representing an attempt to reproduce the past[5]. Newtro, a concept derived from retro, is a new term that combines 'new' and 'retro,' and denotes the tendency to reinterpret the concept of retro. Importantly, the main consumers of 'Newtro' are the young generation, who have never experienced the past. Lee Yun-jung (2019) said that the young generation, who have lived without material deficits, are mentally satisfied with the charm of Newtro's imperfections in a highly civilized society[3]. Kim Min-hyung (2019) argued that the young generation, who have used various digital devices and electronic products, feel a temporary sense of freedom from 'digital fatigue' by encountering Newtro. There is also a negative opinion, i.e., that the young generation is feeling helpless against the convenience of technology, and is looking for Newtro as a refuge from reality[6]. Therefore, Newtro has three sociocultural characteristics: unfamiliarity and novelty, psychological deficits, and a sense of stability. The first characteristic of Newtro is that the retro, which was popular 20-30 years ago, is perceived as 'unfamiliar and new' by young people who have never lived in the past. Digital natives, i.e., a generation born in a digital environment and using digital devices in their daily life, have a desire to escape from the fatigue of reality, and not to a convenient digital world. Therefore, the second characteristic of Newtro is that it plays a role in breaking out of the 'psychological deficit' that arises from digital fatigue. The third characteristic is that the young generation feels more 'mental satisfaction and comfort' in the old, uncomfortable, and incomplete than in the perfect and splendid modern life. This is because the young generation of the modern age lives in a materially affluent environment, but also in a mentally deficient environment. In summary, retro has the characteristic that generations who have experienced the past feel nostalgia from the old; Newtro has the characteristic that generations who have not experienced the past feel new from the old [6-8].

Interactivity is a physical and psychological phenomenon that occurs between humans and media, and between humans and humans[1]. In human-computer interaction(HCI), interaction refers to the coexistence of humans and computers[9]. Since the early 1980s, interaction has begun to attract attention in new media communication, and most of today's content, where the convergence of various fields is active, has a characteristic of interaction $[1,10]$. The physical interactions, mainly appearing in the new media, are perceptual experiences obtained by directly using the human senses (i.e., sensing with the five senses through a physical medium). Among the new media contents, interactive content comprises the addition of interactions to multimedia content along with the development of information and communications technology, enabling user interaction with the content[11]. For interaction to occur, a user interface connecting users and contents is required. Interfaces generally refer to the faces of two different worlds, which act as the contact points between people, tools, and machines. Modern interfaces provide the user with a computer environment, and users need only use the environment provided by the computer. User interfaces also provide a way for humans and content to 'communicate.' In other words, the interfaces serve to help the user and computer (content) to interact. Therefore, the interface plays an important role in active interactions between users and contents[2, 13].

Based on the contents summarized in Chapter 2, a study was conducted using interactive content, converging art and technology. In view of Newtro's sociocultural background, there was an attempt to relieve the digital fatigue experienced by the young generation living in the modern age using Newtro. Newtro was applied to the contents by adding a modern element of interaction to the contents using objects representing the retro concept. In addition, a study was conducted on the physical interactions occurring for the interactive content, and the interface between the users and content. First, to derive a way to increase the level of immersion in the content, interactive content was considered. Furthermore, an interface requiring physical force was used, and a study was conducted to determine whether the sense of presence regarding the content could be enhanced through such an interface. A research direction was determined for interactive content in terms of the physical interaction and user interface.

\section{Interactive content using hand interface}

In the process of visualizing the content, the visual concept of the overall content was decided based on inspiration from Newtro. The retro characteristic was maximized by using representative objects of the retro concept, i.e., an LP and a turntable. In addition, background music, one of the important elements of the content, used music that remixed modern music into the style of the 1990s. In other words, Newtro, which added modern characteristics to retro, was produced as the interactive content. As shown in Figure 1, in the technology structure used for this content, the user pressed a button connected to an Arduino board, and a video from the PC was played back to a screen through a projector. For the immediate interaction via stimulus and reaction (one of the main characteristics of interactive content), a physical interaction that the user could directly sense was used. To apply physical interaction to interactive content, a button interface to which a user's physical force is applied was used. The button interface was designed in the form of a DJ box, according to the concept of Newtro. As shown in Figure 2, the DJ box was designed with the Rhino CAD program and was printed with a 3D printer. A 
button interface was used because the user is required to use greater physical force, and this physical action allows the user to feel a presence regarding the content.

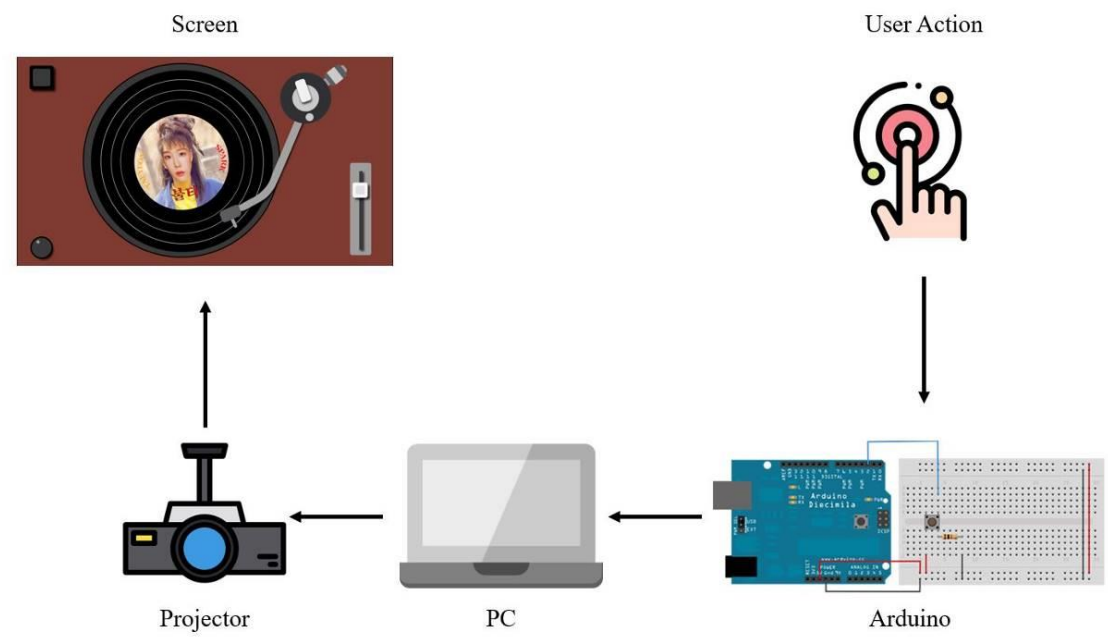

Figure 1 System structure

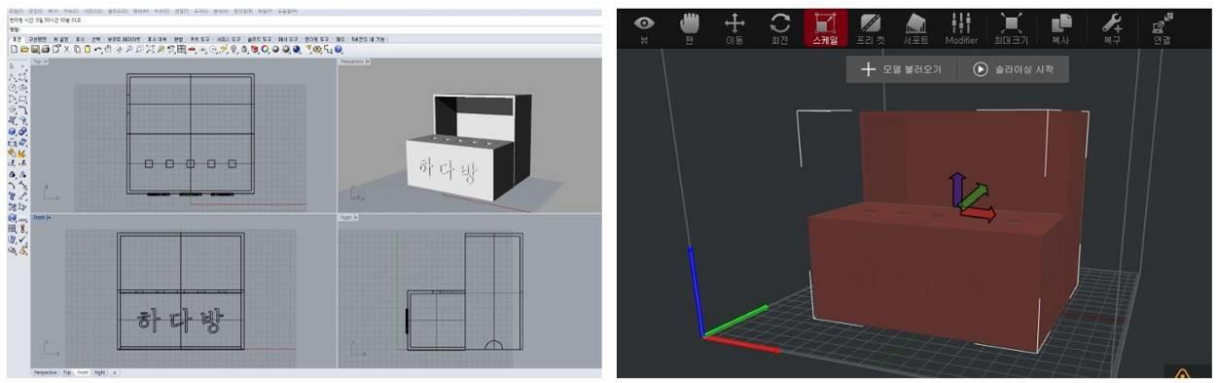

Figure 2 DJ box modeling

There were a total of five buttons installed in the DJ box, and each time the button was pressed, different five video clips were played. Because buttons were used, the input values of each button were set through Arduino programming, and the Arduino board was installed in the DJ box as shown in Figure 3. Through serial communication between the Arduino board and the PC, the code for each video played in the Unity software of the PC was generated. When one video finished, the system automatically returned to the video set as the initial video.
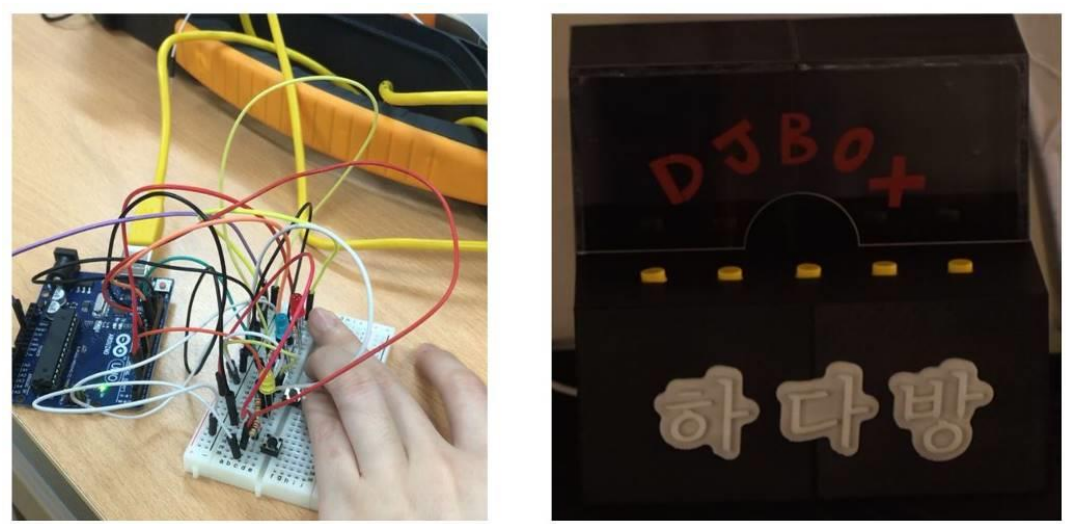

Figure 3 Controller in the DJ box

As shown in Figure 4, before the button was pressed, an initial video with the music list written on the LP was played repeatedly. When the user pressed the five buttons installed on the button case, the different videos were played, as shown in the image on the right. When the video played by pressing the button was finished, the system returned to the initially set video. 


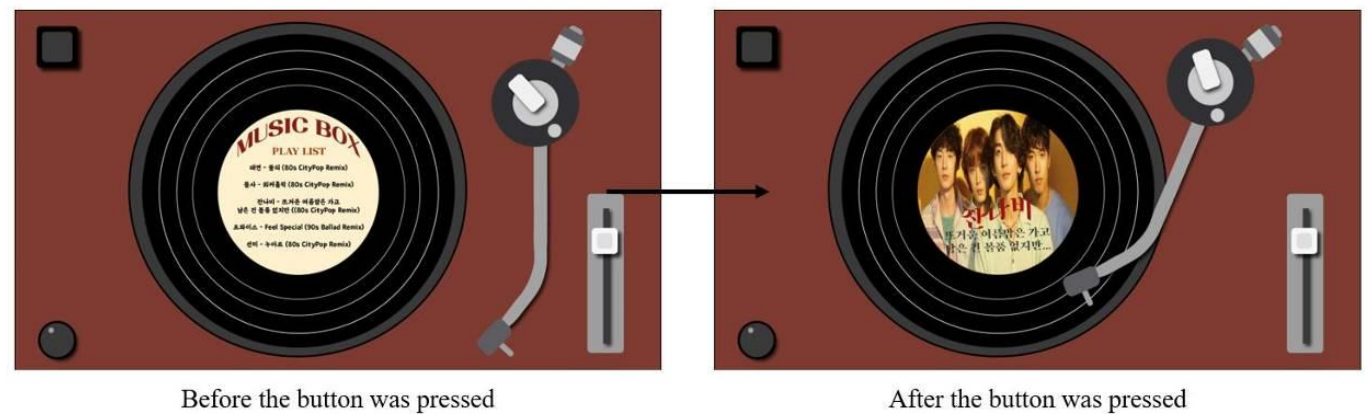

Figure 4 Interactive video

\section{Conclusions}

As shown in Figure 5, in the interactive content proposed in this study, an interaction in which a video was played when a user pressed a button was used. The interactions (i.e., the physical interaction) between the user and content were discussed. To apply the physical interaction to the content, an interface for applying physical force was discussed, and a button interface was used to facilitate the physical interaction more easily.

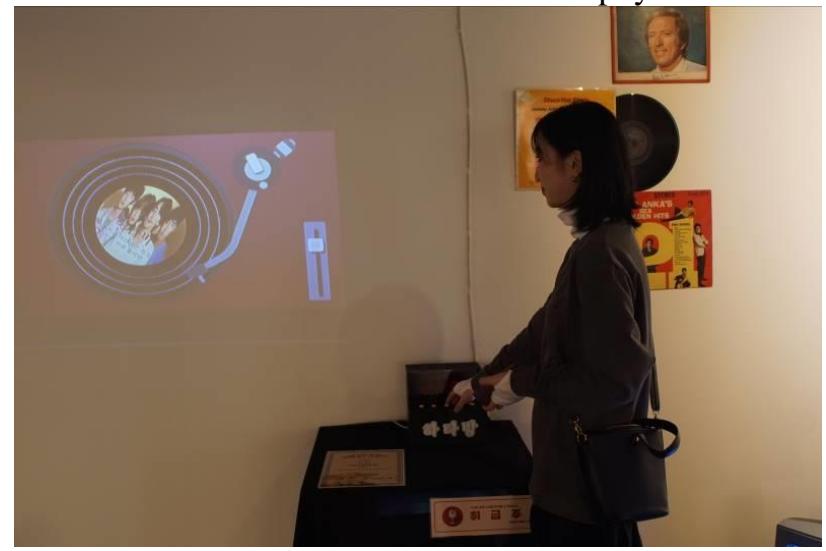

Figure 5 Interactive content presentation

A survey was conducted with 60 users who experienced the content. The survey consisted of a total of five questions, and based on the produced content, the questions were set to meet the objectives of the study. The focus of this research was to investigate whether content with interaction enhances the immersiveness in communications between users and content. It was investigated whether the proposed button interface was helpful in the interactions between content and user. In addition, it was investigated whether the feeling of presence in the content was higher when a button interface requiring greater physical force than, e.g., a touch sensor requiring relatively little physical force was used. Finally, it was investigated whether the button interface, which requires relatively greater physical force, was helpful for the physical interaction. The results of the survey were analyzed, as shown in Figure 6. The horizontal axis of the graph shows how the users feel regarding the content, and the vertical axis shows the number of people. As shown in the graph of Figure 6-(a), the responses to the question "Do you think that content that allows users to interact with users can increase immersion in content rather than content in the form of passive viewing of content?" were as follows: Strongly Agree - 41 persons (68.3\%), Agree - 17 persons (28.3\%), Neutral - 1 person (1.7\%) Disagree - 0 persons, Strongly Disagree - 1 person $(1.7 \%)$. As shown in the graph of Figure 6-(b), the responses to the question "Do you think the 'button interface' used for this content was helpful for interaction with the content?" were as follows: Strongly Agree - 31 persons (51.7\%), Agree - 23 persons (38.3\%), Neutral - 5 persons (8.3\%), Disagree - 0 persons, and Strongly Disagree - 1 person (1.7\%). As shown in the graph of Figure 6-(c), the responses to the question "Did you feel more presence about the content when you used the 'button interface' that requires more physical force than the interface where the user's physical force is relatively less applied?" were as follows: Strongly Agree - 30 persons (50\%), Agree - 20 persons (33.3\%), Neutral - 6 persons (10\%), Disagree - 3 persons (5\%), and Strongly Disagree - 1 person (1.7\%). As shown in the graph of Figure 6-(d), the responses to the question 'Do you think the 'button interface', which requires a relatively more physical force, is helpful for physical interaction?" were as follows: Strongly Agree - 34 persons (56.7\%), Agree - 16 persons (26.7\%), Neutral - 5 persons (8.3\%), Disagree - 4 persons (6.7\%), Strongly Disagree - 1 person (1.7\%). 


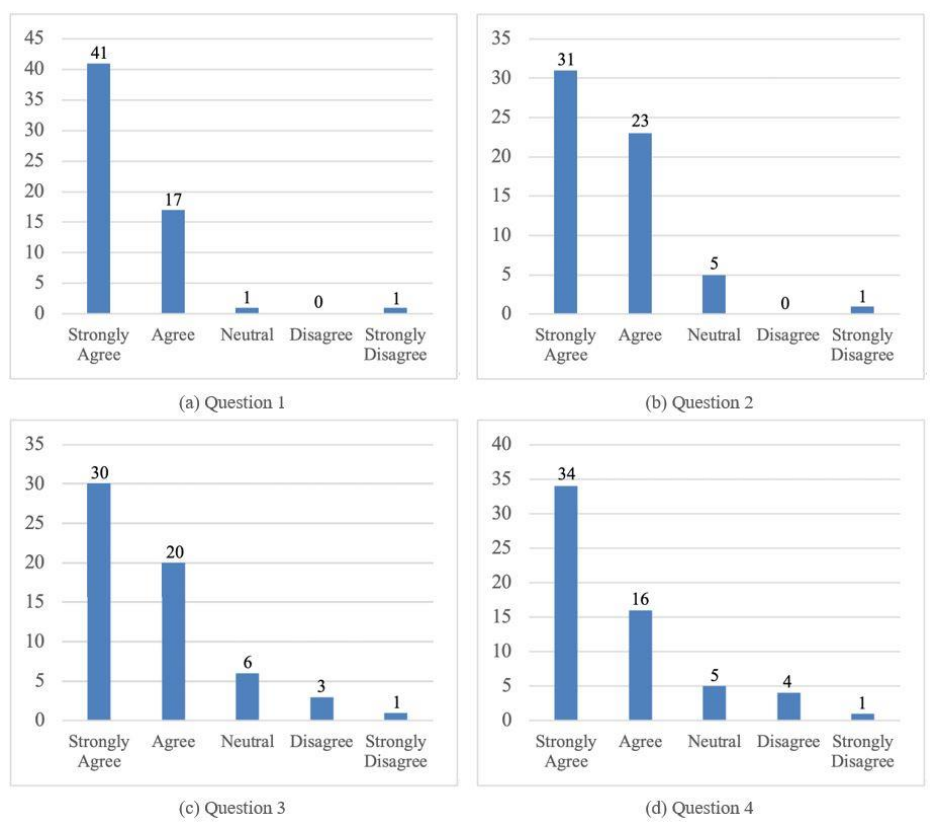

Figure 6 Survey results

Based on the analysis of survey results, the conclusions of the study were determined. It was observed that interactive content is helpful for the interaction between users and content, and such interaction can increase the immersion in the content. In addition, a 'button interface' that requires relatively greater force allows the user to feel a great sense of presence for the content. The results show that the degrees of interaction that the user feels when experiencing the content are different depending on the degree of physical force of the interface, and if the user actively applies a greater force, they can feel a higher presence in the content. This study is significant in that it analyzes the sociocultural phenomenon of Newtro, and treats it as interactive content through a convergence of technology and art. In addition, it is meaningful in that the physical interaction between the user and content is discussed, and content is produced with an interface that relatively requires greater physical force from the user. In that regard, it was meaningful that the button interface was helpful for physical interaction; however, as a relatively simple technology was used, it could somewhat reduce the user's interest in the content. Therefore, further study is required on a more active communication method for the interactions between content and users, and on ways to further increase the user's interest and immersiveness in the content.

\section{Acknowledgements}

This research was supported by Basic Science Research Program through the National Research Foundation of Korea(NRF) funded by the Ministry of Education(NRF-2020R1I1A3051739) and Institute of Information \& communications Technology Planning \& Evaluation(IITP) grant funded by the Korea government(MSIT)(No.2020-0-00238, Virtual Reality Force Feedback Controller Based User Biosignal Interface and Contents Control Technology)

\section{References}

1. Lee Joo-Hee. Interactivity of New-media Contents with Cultural Semiotics Analysis. Journal of Digital Contents Society. 2020;21(3):505-512.

2. Hong Jong-Iin. A Study on Interface Design based on Understanding User Interface. In Proceedings of the Korea Contents Association Conference; 2004.

3. Yuh Hea-Min, Son Won-Jun. Analysis of Cycle Characteristics of Retro Design Trend -Consumer Trends and Visual Cases Focused on Can Design -. Korea Society of Design Trend; 2019. p. 239-248.

4. Nam Mee-Kyung. A Study on New Retro Design Utilized by Combining Emotion and Newtechnology. JOURNAL OF THE KOREAN SOCIETY DESIGN CULTURE. 2018;24(2):205-213.

5. Lee Yun-Jung. A Study on the Formative Characteristics of Package Design Reflecting New-tro Design. Brand Design Association Of Korea; 2019. p. 85-98.

6. Kim Min-Hyeong, Jeon Ha-Min, Kim Dong-Young. Newtro Trend. Korea Marketing Association; 2019. p. 57-65.

7. Park Song-Ae. A study on the phenomenon of new-tro expressed in fashion - Focus on music video costume style -. Journal of the Korea Fashion \& Costume Design Association. 2019;21(3):137-147.

8. Kim Nan-Do. Trend Korea. Seoul: Miraebook. 2019.

9. Cleotilde G. Visual design of interaction, dialog, or interface?. SIGCHI Bulletin; 1995. p. 12-13. 
10. Kim Byoung-Cheol. A Study on the Effects of Interactivity on Discussion by Internet Newspaper Users. Journal of Cybercommunication Academic Society. 2004;(14):147-180.

11. Catherine B. Interactivity and immersion in a media-based performance. journal of audience \& reception studies. 2014;11(1):254-269.

12. Hong Dong-Pyo, Woo Woon-Tack. Recent Research Trend of Gesture-based User Interfaces. Telecommunications Review; 2008. p. 403-413. 